\title{
Investigación en Medicina Familiar y Comunitaria en Iberoamérica
}

\section{Research in Family and Community Medicine in Ibero-America \\ Pesquisa em Medicina de Família e Comunidade na Ibero-América}

Noris Margarita Serrudo de Domínguez. Gobernación del Estado Zulia Secretaria de salud; Secretaría de Finanzas del Capitulo Zulia; Sociedad Venezolana de Medicina Familiar (SOVEMEFA); Subregión Países Andinos de la Confederacion Iberoamericana de Medicina Familiar, (Wonca-Iberoamericana-CIMF); Centro de Medicina Familiar Padilla. Venezuela. norisserrudo@gmail.com (Autor correspondiente) Jacqueline Ponzo. Universidad de la República, Facultad de Medicina (UDELAR); Unidad Docente Asistencial Canelones al Este; Red IBIMEFA, Wonca Iberoamericana-CIMF. Uruguay. ponzo4@gmail.com (Autora correspondiente) José Manuel Ramírez Aranda. Universidad Autónoma de Nuevo León (UANL); Hospital Universitario “José Eleuterio González"; Red Mexicana de Investigadores en Medicina Familiar AC. México. sersabe2010@gmail.com

Carla Alexandra Argudo Haro. Centro de Salud Familiar San Joaquín, Departamento de APS y Salud Familiar Universidad de Chile; Facultad Medicina Catedra Residentes Medicina Familiar Tutoria clínica. Ecuador. carliarg@yahoo.com Miriam Elisa Riveros Ríos. Facultad de Ciencias Medicas, Universidad Nacional de Asunción; Cátedra de Medicina Familiar Filial Santa Rosa. Paraguay. melisarr76@ hotmail.com

Pablo Vargas Villarreal. Caja Costarricense del Seguro Social; Universidad de Costa Rica. Costa Rica. pavavi@gmail.com Juan Víctor Ariel Franco. Servicio de Medicina Familiar y Comunitaria del Hospital Italiano de Buenos Aires; Investigador Centro Cochrane - Instituto Universitario del Hospital Italiano de Buenos Aires; Cátedra de Salud Pública; Universidad de Buenos Aires. Argentina. juan.franco@ @ospitalitaliano.org.ar Sergio Adrián Terrasa. Servicio de Medicina Familiar y Comunitaria y Departamento de Investigación del Hospital Italiano de Buenos Aires; Departamento de Salud Pública del Instituto Universitario Hospital Italiano. Argentina. sergio.terrasa @ hiba.org.ar Juan Carlos Perozo García. Universidad Nacional Experimental Francisco de Miranda (UNEFM); Departamento de Integración Docente Asistencial, centro de Investigaciones Biomédicas de la (UNEFM); Sociedad Venezolana de Medicina Familiar (SOVEMEFA). Venezuela. jucape33@ gmail.com María Cristina da Costa Días. Sociedad Venezolana de Medicina Familiar (SOVEMEFA); Ambulatorio Militar La Rosaleda. Venezuela. cristydacosta@yahoo.com

Fausto Gady Torres Toala. Makroscopio Servicios de Salud Cia Ltda; Universidad Católica del Ecuador. Ecuador. gtorres@makroscopio.com Roberto Flete González. Hospital General Engombe. República Dominicana. robertoflete@ gmail.com - fleteroberto@ hotmail.com

\section{Grupo de trabajo}

Abril Collado RO (Perú); Avila A (Colombia); Bermúdez GA (Venezuela); Caballero L (Argentina); Cardozo de Angulo RA (Venezuela); Colon González MC (Puerto Rico); Cordero Tapia PA (Bolivia); da Silva AL (Brasil); Domínguez Serrudo NV (Venezuela); Ehlerman Escalante CC (El Salvador); Esteban S (Argentina); Gavilanes Cueva YP (Ecuador); Germosen Almonte YR (República Dominicana); Gloria Cristina Cordoba Currea GC (Colombia); Homero de los Santos Reséndiz H (México); Karen Vanessa Muñoz Chamorro KV (Colombia); Luna-Ruiz MA (México); Martinez-Bianchi V (Argentina); Meaux JA (Puerto Rico); Mejía M (Venezuela); Morón Vásquez A (Venezuela); Munive Angermuller M (Costa Rica); Olinisky Bentancor MM (Uruguay); Patricia Elizabeth Hernández Coronado PE (Bolivia); Paulo A (Uruguay); Paz NA (Honduras); Piñol Moreso JL (España); Ramirez Nizza R (Paraguay); Rava Dellepiane P (Uruguay); Rivera P (Panamá); Rodríguez Escobar MA (Colombia); Rojas Velasco GW (Ecuador); Trindade TG (Brasil); Vietto V (Argentina)

\section{Financiación:}

ninguna declarada

Aprobación ética

El siguiente trabajo no cuadra como investigación sobre

sujetos humanos sino constituye un relevamiento como parte de un proceso organizativo de la red IBIMEFA. Implica bajo riesgo ético, sin embargo los autores declaran su adherencia a los principios éticos de la Asociación Médica Mundial y la Declaración de Helsinki. Conflicto de intereses: ninguna declarada.

Procedencia y revisión por pares: revisado por pares. Recibido el: 15/08/2016. Aceptado el: 15/09/2016. 


\section{Resumen}

El grupo de trabajo \# 4 de la VI Cumbre Iberoamericana de Medicina Familiar en San José, Costa Rica, 2016 se conformó para lograr contribuir al desarrollo de la investigación en Medicina Familiar y Atención Primaria en Iberoamérica, así como para consolidar la Red IBIMEFA. Estuvo integrado por 54 médicos de 21 países. Se nombraron 2 coordinadores generales y 10 coordinadores para dirigir los cinco subgrupos. El trabajo realizado por este grupo durante ocho meses, obtuvo como resultado la identificación de líneas activas y prioritarias de investigación en medicina familiar, la necesidad de realizar estrategias para promover la producción científica, tales como: a) desarrollo de pasantías de investigación en diferentes regiones; b) identificación de fuentes de financiamiento; c) lograr una plataforma virtual, soporte para asesorías y foros de investigación coordinados por IBIMEFA.

\section{Abstract}

In order to contribute to the development of research in Family Medicine and Primary Care in Ibero-America and the consolidation of the IBIMEFA Network, the Work Group \#4 was created for the VI Ibero-American Summit of Family Medicine in San José, Costa Rica, 2016, which was composed by a group of 54 family physicians from 21 countries. Two general coordinators and 10 subgroup coordinators were designated. The work developed by this group throughout an 8-month period has resulted in the identification of both active and priority research lines in Family Medicine and the need to develop strategies for the promotion of scientific production, such as: a) the development of research internships across the different regions, b) the identification of sources of financing; c) the design of a virtual platform with support for consultancy and research forums coordinated by IBIMEFA.

\section{Resumo}

O grupo de trabalho ํㅜ 4 da VI Cúpula Ibero-Americana de Medicina Familiar em San Jose, Costa Rica de 2016 se organizou para colaborar com o desenvolvimento da pesquisa em Medicina de Família e Atenção Primaria na Ibero-América, assim como consolidar a Rede IBIMEFA. Foi composto por 54 médicos de 21 países. Dois coordenadores gerais e 10 sub-coordenadores foram nomeados para organizar os cinco subgrupos. $O$ trabalho realizado durante oito meses por este grupo obteve como resultado a identificação de linhas ativas e prioritárias de pesquisa em Medicina de Família e a necessidade de serem estabelecidas estratégias para promover a produção científica tais como: a) implementação de estágios de pesquisa em diferentes regiões; b) identificação de fontes de financiamento; $c$ ) desenvolver uma plataforma virtual, dar suporte para consultoria e realizar fóruns de pesquisa coordenados pela IBIMEFA.
Palabras clave:

Investigación

Medicina Familiar

Atención primaria

IBIMEFA

\section{Keywords:}

Research

Family medicine

Primary care

IBIMEFA

\section{Introducción}

En la historia del trabajo organizado desde la Confederación Iberoamericana de Medicina Familiar (CIMF) se pueden identificar varios momentos relevantes para promover la investigación en la región: el Primer Taller de Investigación en Medicina Familiar, ${ }^{1}$ realizado en Cali (Colombia, 2008) tomó las recomendaciones de la reunión de Ontario ${ }^{2}$ de The World Organization of Family Doctors (WONCA) para el impulso de la investigación en medicina familiar y atención primaria e instaló la Red Iberoamericana de Investigación en Medicina Familiar (IBIMEFA). ${ }^{3}$ En la IV Cumbre Iberoamericana de Medicina Familiar (2011, Asunción, Paraguay), el trabajo del Grupo de Investigación emitió un documento de diagnóstico que sintetizaba las dificultades, oportunidades, desafíos y algunas recomendaciones para lograr el avance. ${ }^{4}$ La Carta de Quito -resultado de la V Cumbre Iberoamericana-, recomienda promover la investigación como elemento fundamental para mantener el nivel profesional de los médicos de familia. ${ }^{5}$ En 2015 tuvo lugar en Montevideo el Segundo Taller Iberoamericano de Investigación en Medicina Familiar y Atención Primaria y la primera reunión de editores de revistas de medicina familiar y comunitaria (MFYC) de la región.

El presente documento se produjo en el marco de la VI Cumbre Iberoamericana de Medicina Familiar y Comunitaria (San José, Costa Rica, 2016) y da continuidad a los procesos reseñados. Su producción se inscribe en el desarrollo de la Red IBIMEFA, en tanto contribuye a la vinculación entre investigadores, promueve el involucramiento de las Asociaciones miembro de CIMF con la investigación y aporta elementos de valor para la planificación estratégica de la investigación en la Iberoamérica. El objetivo del trabajo es contribuir al desarrollo de la investigación en Medicina 
Familiar y Atención Primaria en Iberoamérica y a la consolidación de la Red IBIMEFA como herramienta para la acción colaborativa permanente.

\section{Métodos}

Se trabajó en un grupo abierto, instalado a partir de la convocatoria realizada por el Comité Ejecutivo de CIMF a las Asociaciones miembro y el Movimiento de Jóvenes Médicos de Familia (Waynakay). Se integraron al grupo las personas nominadas por los referentes de estas Asociaciones, así como también Médicos de Familia externos a la región, pero vinculados a Iberoamérica, que respondieron a la convocatoria (Estados Unidos y Dinamarca). También se dio lugar a la integración de médicos de países que actualmente no son miembros de CIMF, tal el caso de Honduras y Nicaragua, en favor de promover el desarrollo de la MFYC en estos países y su integración a la Confederación. Una vez instalado el Grupo de Trabajo, se identificaron las líneas prioritarias para el desarrollo de la investigación y con base en a ellas se establecieron los objetivos y se instalaron cinco subgrupos. El proceso de trabajo se extendió durante ocho meses (septiembre 2015 - abril 2016) con la participación de 54 médicos de 21 países. Se realizaron 23 reuniones virtuales e intercambio vía e-mail, con elaboración colaborativa de documentos. Cumplida la primera etapa de trabajo en subgrupos; se realizó en forma integrada el análisis de los resultados y la elaboración de conclusiones y recomendaciones. Durante la VI Cumbre Iberoamericana de MFYC realizada en San José, se desarrolló un taller que profundizó en algunos aspectos del documento preliminar. De esta fase final, se destaca la participación de jóvenes médicos de familia del Movimiento Waynakay.

En el cuadro 1 se resumen los métodos y fuentes de datos para cada objetivo de trabajo.

Cuadro 1. Objetivos del documento en los subgrupos de trabajo.

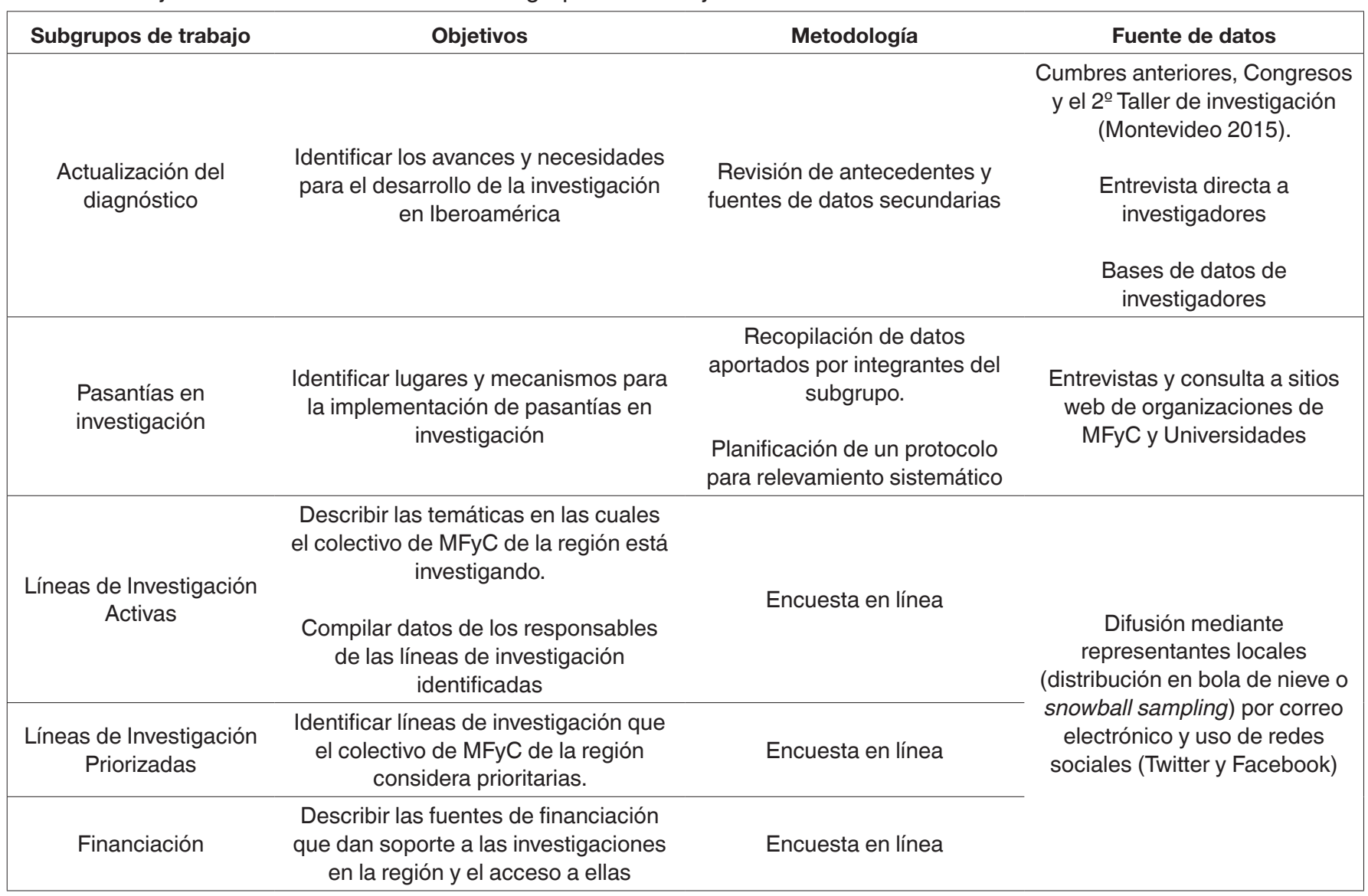




\section{Resultados}

\section{Situación Iberoamericana}

La necesidad de fortalecer la investigación a través de las redes fue lo que desencadenó la reunión de WONCA 2003. En la reunión de Cali, Colombia (2008), con la creación de la IBIMEFA, se señaló que el incremento en las competencias metodológicas para la investigación. La generación de incentivos y la creación de maestrías en Medicina Familiar y Atención Primaria era prioritario, pero no se avanzó en los mecanismos para concretar esto.

En la III Cumbre Iberoamericana (Fortaleza, Brasil), ${ }^{6}$ se ratificaron los acuerdos de Cali y se señaló la importancia de la difusión de conocimiento en la región como otra estrategia que contribuye al desarrollo académico.

En la IV Cumbre Iberoamericana (Asunción, Paraguay), ${ }^{7}$ se planteó a la investigación como eje estratégico para el desarrollo de sistemas de salud más eficientes y equitativos. Por otra parte, se expresó la necesidad de la mentoría en la investigación, pero sin determinar en ese momento los mecanismos para llevarla a cabo en las diferentes instituciones donde se realizan investigaciones. También se hizo mención a la pertinencia de manejar una agenda común de investigación para todos los países de Iberoamérica, así como la importancia de que los resultados de dichas investigaciones sean comunicados a los decisores.

En la V Cumbre Iberoamericana (Quito, Ecuador), ${ }^{8}$ se destacó la importancia de potenciar el uso de las tecnologías en la información y comunicación para la investigación. Sin embargo, el soporte informático de CIMF aún es limitado y se requiere ampliar las estrategias o líneas de acción para responder a este enunciado. En 2014, las líneas de investigación activas derivadas de una encuesta en la que participaron 13 países fueron: enfermedades crónicas degenerativas (diabetes mellitus, hipertensión arterial, enfermedad renal crónica), organización de servicios de salud, evaluación de servicios de salud, salud familiar, salud comunitaria, determinantes sociales, educación para la salud y adulto mayor.

En el 2do. Taller Iberoamericano de Medicina Familiar y Atención Primaria realizado en Montevideo (Uruguay), ${ }^{9}$ en el marco del 4to. Congreso Iberoamericano de CIMF, se realizó un relevamiento y se inició un registro de investigadores en la región que constituye el insumo inicial para una base de datos necesaria. Mediante un formulario online, se alcanzó un registro de 97 investigadores con una edad promedio de 45 años (DS 12), 65\% mujeres y 65\% con nivel académico de maestría o doctorado ( $80 \%$ ha completado algún curso formal de metodología de la investigación). Participaron 15 países, quienes opinaron sobre las áreas de interés en investigación, se destacaron: la orientación comunitaria, las enfermedades crónico-degenerativas, la efectividad de los métodos educativos, competencias en la resolución de problemas clínicos, organización de servicios de salud, enfoque preventivo y cuidado del adulto mayor. Se establecieron lineamientos para cumplir a mediano y largo plazo:

a) desarrollo de pasantías de investigación en diferentes regiones y países, como forma de promover el intercambio, la coordinación y la producción científica;

b) identificación de fuentes de financiación que permitan fortalecer y llevar a cabo las líneas de investigación necesarias;

c) lograr una plataforma virtual, soporte para asesorías y foros de investigación coordinados por IBIMEFA, que idealmente debiera estar radicado en el sitio web de WONCA Iberoamericana-CIMF.

\section{Líneas de investigación activas en Iberoamérica}

Se obtuvo 86 entradas al formulario de encuesta en línea desde el 22 de octubre hasta el 23 de noviembre de 2015, con representación de 16 países.

Se encontró que 16\% de las líneas de investigación activas eran de carácter metodológico; es decir, orientadas al desarrollo y perfeccionamiento de ciertas actividades (por ejemplo: diseño de guías de práctica clínica para el 
primer nivel de atención), en tanto que $84 \%$ restante estaban orientadas a problemas de la práctica de la MFYC (ej.: abordaje desde el primer nivel de atención de la violencia de género).

Un 49\% informaron contar con financiamiento para las líneas de investigación mencionadas.

De las líneas de investigación activas reportadas, 15 de ellas (17\%) no tenían trabajos de investigación en curso, 24 de ellas (28\%) tenían trabajos en desarrollo y las 47 restantes tenían trabajos de investigación terminados.

En el cuadro 2 se puede ver la lista de líneas de investigación activas y sus países.

Cuadro 2. Líneas de investigación en Medicina Familiar y Comunitaria activas en Iberoamérica. Abril 2016.

\begin{tabular}{|c|c|}
\hline Nombre de la línea de investigación & País \\
\hline Abordaje al Tabaquismo & España \\
\hline Actividad Física y Salud & España \\
\hline Adaptaciones y Armonización de versiones de PCAT en Iberoaméricar & Red Iberoamericana PCAT \\
\hline Análisis de consultas no urgentes en el servicio de emergencias & Costa Rica \\
\hline Atención a la Familia & España \\
\hline Atención a la Mujer & España \\
\hline Atención al Adolescente & España \\
\hline Atención al Mayor & España \\
\hline Bioética & España \\
\hline Calidad de vida & Venezuela \\
\hline Calidad de vida relacionada con la salud en mujeres de edad mediana & Cuba \\
\hline Calidad y seguridad del paciente & México \\
\hline Cáncer & España \\
\hline Cirugía Menor y Dermatología & España \\
\hline Consulta integral posaborto u óbito & Costa Rica \\
\hline Cuidados Paliativos & España \\
\hline Demencias & España \\
\hline Dependencia Severa & Chile \\
\hline Diabetes & España \\
\hline Dislipemias & España \\
\hline Diversidad Sexual y Vejez & Uruguay \\
\hline Ecografía & España \\
\hline Eficacia de la entrevista motivacional en el manejo de la obesidad & Panamá \\
\hline Enfermedades crónicas no transmisibles & Colombia \\
\hline Enfermedades Cardiovasculares & España \\
\hline Enfermedades crónicas no transmisibles & Venezuela \\
\hline Enfermedades Infecciosas & España \\
\hline Enfermedades Infecto-Contagiosas & Venezuela \\
\hline Enfermedades Respiratorias & España \\
\hline Enfermedades Reumatológicas & España \\
\hline Enfoque de riesgo biopsicosocial en adultos & Venezuela \\
\hline Enfoque de riesgo biopsicosocial en niños y adolescentes & Venezuela \\
\hline Estudio de Leptospirosis en el Entorno de Grupos Humanos en Riesgo & Uruguay \\
\hline $\begin{array}{l}\text { Estudio de seguimiento de factores de riesgo y enfermedades cardiovascultares en el Cono Sur de } \\
\text { América }\end{array}$ & Uruguay \\
\hline $\begin{array}{l}\text { Evaluación de Calidad de Vida del Diabético Tipo-2 Clínica de Diabetes Policlínica Hugo-Spadafora } \\
\text { Abril-Mayo } 2013\end{array}$ & Panamá \\
\hline
\end{tabular}




\section{Continuación Cuadro 2.}

\begin{tabular}{|c|c|}
\hline Nombre de la línea de investigación & País \\
\hline $\begin{array}{l}\text { Evaluación de la Formación Basada en Competencias de los Residentes del Programa de la } \\
\text { Especialidad en Medicina Familiar CHMDRAAM - Facultad de Medicina - Universidad de Panamá }\end{array}$ & Panamá \\
\hline $\begin{array}{l}\text { Evaluación de la Metas de Control Metabólicas Pacientes de Medicina Familiar Clínica de Diabetes } \\
\text { Policentro de Parque Lefevre 2010-2011 }\end{array}$ & Panamá \\
\hline Factores de riesgo & El Salvador \\
\hline Familia & El Salvador \\
\hline Formación de Talento Humano en Medicina Familiar en la región & Colombia \\
\hline Genética Clínica y Enfermedades Raras & España \\
\hline GIEMFAPS. Grupo de Investigación Estratégica en Medicina de Familia y Atención Primaria de Salud. & Venezuela \\
\hline $\begin{array}{l}\text { Grado de cumplimiento de objetivos terapéuticos en pacientes con diabetes mellitus tipo } 2 \text { del servicio } \\
\text { de MFYC de Unidad Docente Asistencial (UDA) Saint Bois }\end{array}$ & Uruguay \\
\hline Hipertensión Arterial & España \\
\hline International Collaboration to Promote Scholarly Activity Among Young Family Physicians & Colombia \\
\hline Intervención en Drogas & España \\
\hline Intervenciones educativas en salud & Venezuela \\
\hline Manejo Terapéutico & El Salvador \\
\hline Medicina Basada en la Evidencia & España \\
\hline Medicina Familiar como Estrategia de Gestión de la Calidad del Sistema de Salud & Ecuador \\
\hline Medicina Familiar y Comunitaria & Venezuela \\
\hline Medicina Familiar y Comunitaria & Venezuela \\
\hline Medicina Familiar y Salud Pública & Venezuela \\
\hline $\begin{array}{l}\text { Medicina Herbolaria en pacientes Comórbidos de Medicina Familiar en Policlínica Presidente Remón. } \\
\text { OCTUBRE - DICIEMBRE } 2014\end{array}$ & Panamá \\
\hline Medicina Rural & España \\
\hline Medidas educativas para cuidadores del hospital geriátrico & Paraguay \\
\hline Multimorbidade e seu impacto nos sistemas de saúde & Brasil \\
\hline Nefrourología & España \\
\hline Neurología & España \\
\hline Nutrición y Alimentación & España \\
\hline $\begin{array}{l}\text { Orientación hacia la Atención Primaria de la Salud (APS) como estrategia para mejorar la calidad de } \\
\text { atención en el primer nivel }\end{array}$ & Argentina \\
\hline Prevención cuaternaria & Perú, Argentina \\
\hline Primary Care Assessment Tool - PCAT. Adaptación del instrumento y evaluación de servicios de salud & $\begin{array}{l}\text { Argentina, Bolivia, Brasil, } \\
\text { Uruguay }\end{array}$ \\
\hline Procedimientos en Atención Primaria & El Salvador \\
\hline $\begin{array}{l}\text { Programas formativos para el equipo de salud en educación para pacientes. Programas de educación } \\
\text { para pacientes y sus familiares con enfermedad crónica }\end{array}$ & Venezuela \\
\hline Referencia y contrarreferencia en servicios de salud & Argentina \\
\hline Riesgos en salud/Formación en APS & Venezuela \\
\hline Salud Basada en las Emociones & España \\
\hline Salud Familiar y Atención Primaria & Colombia \\
\hline Salud Mental & España \\
\hline Salud pública y medicina comunitaria & Colombia \\
\hline Salud Sexual y Reproductiva & Venezuela \\
\hline Seguridad del Paciente & España \\
\hline
\end{tabular}




\section{Continuación Cuadro 2.}

\begin{tabular}{|lc|}
\hline Nombre de la línea de investigación & País \\
\hline Tamizaje & El Salvador \\
Toma de decisiones compartidas y medicina centrada en las personas & Argentina \\
Toxicología y Salud Ocupacional & Venezuela \\
& Marcelo Salinas Rojas \\
Transdisciplinariedad y APS & salinasmarc99@gmail.com \\
& (Investigador Independiente) \\
Transferencia del conocimiento (Knowledge translation) & Argentina \\
Urgencias y Atención Continuada & España \\
Uso de antibióticos en atención primaria. HAPPY AUDIT II Sudamérica & Argentina, Bolivia, Paraguay, \\
Uso de la escala de Edimburgo para la pesquisa de riesgo de depresión post parto de las puérperas & Uruguay y Dinamarca \\
que tuvieron su parto en el departamento de Florida. & Uruguay \\
Utilización de Fármacos & España \\
VIH & España \\
\hline
\end{tabular}

\section{Líneas de investigación consideradas prioritarias}

Hubo 114 participantes de la encuesta, de la cual se obtuvieron 107 respuestas satisfactorias y siete incompletas o incorrectamente llenadas que no fueron tomadas en cuenta. La mayor parte de los encuestados que respondieron esta consulta no tenía líneas de investigación activas (64\%) ni conocía la red IBIMEFA (60\%).

Las prioridades identificadas se distribuyen en tres fracciones similares, aunque con un leve predominio del interés por la investigación clínica (31\%), particularmente los problemas crónicos que por si solos constituyen un $15 \%$ del total. Las otras fracciones son: investigación en sistemas de salud (28\%), particularmente en evaluación de servicios de salud y Primary Care Assessment Tool (PCAT) (11\%). Finalmente, un 24\% señala como prioridad de investigación la formación en mfyc (Gráfico 1).

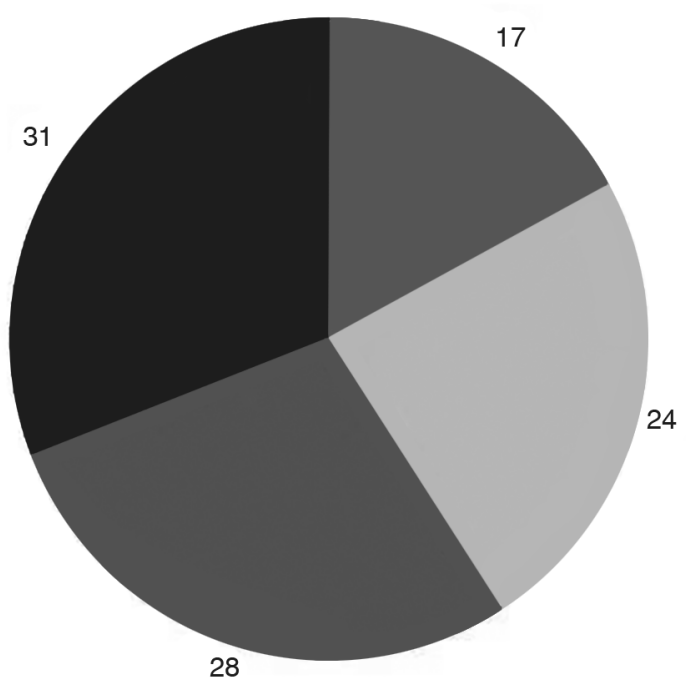

- investigación clínica

investigación en sistemas y servicios de salud

investigación en educación médica/formación en mfyc otras

Gráfico 1. Distribución de las líneas de investigación priorizadas según grandes áreas. 


\section{Comparación entre líneas activas y líneas priorizadas}

Del análisis comparativo entre las líneas identificadas como prioritarias y las líneas activas, se encontró gran coincidencia. Se analizaron las diez líneas que recibieron mayor número de respuestas en favor de su priorización y para cada una de ellas se buscó si se había reportado investigación activa. El resultado de este cruzamiento se puede ver en el cuadro 3. En las diez líneas más priorizadas hay investigación activa en medicina familiar y comunitaria.

Cuadro 3. Comparación entre líneas de investigación activas y priorizadas en lberoamérica, según relevamiento realizado para la VI Cumbre Iberoamericana de Medicina Familiar y Comunitaria (abril 2016).

\begin{tabular}{|c|c|c|}
\hline Línea de investigación priorizadas* & $\begin{array}{l}\text { Frecuencia de respuestas a } \\
\text { favor de su priorización }\end{array}$ & $\begin{array}{l}\% \text { de líneas activas que } \\
\text { abarcan la línea propuesta } \\
{\text { (coincidencia) })^{\star *}}^{\text {(coing }}\end{array}$ \\
\hline 1. Clínica sobre Problemas crónicos en el primer nivel de atención & 16 & 14,95 \\
\hline 2. Formación en medicina familiar & 15 & 14,01 \\
\hline 3. Evaluación de servicios de salud & 11 & 10,28 \\
\hline 4. Adecuación y construcción de instrumentos de medicina familiar & 6 & 5,60 \\
\hline 5. Evaluación de tecnologías sanitarias según MBE & 6 & 5,60 \\
\hline 6. Tecnologización de la salud & 5 & 4,67 \\
\hline 7. Estrés y calidad de vida & 5 & 4,67 \\
\hline 8. Medicina familiar en el ámbito rural & 5 & 4,67 \\
\hline 9. Medicina familiar como política de salud & 4 & 3,73 \\
\hline 10. Prevención Cuaternaria & 4 & 3,73 \\
\hline
\end{tabular}

* Se incluyen las diez líneas que recibieron mayor ponderación según número de respuestas a favor; ** Sobre un total de 86 líneas activas identificadas.

La coincidencia encontrada se puede interpretar como un dato de coherencia entre las convicciones de los especialistas de medicina familiar y comunitaria y sus acciones en relación con la investigación.

\section{Oportunidades para pasantías en investigación}

Se elaboró un protocolo como base de un relevamiento inicial, el cual se aspira profundizar y perfeccionar en la continuidad del trabajo con IBIMEFA. Su objetivo fue caracterizar los lugares de pasantías, con base en datos disponibles en la web o cuestionario a instituciones identificadas para la pasantía de investigadores (Cuadro 4).

De los 26 organismos resultantes, 13 fueron universitarios, 10 organizaciones gubernamentales y cuatro no gubernamentales. No se obtuvo este dato en 11 casos.

De las formas de capacitación, no se reportó exclusiva en aula, la formación en ejercicio se reportó para un caso, 18 tendrían ambas modalidades (aula y práctica) y para siete instituciones no se obtuvo el dato.

En cuanto al perfil requerido para aspirar a una pasantía de investigación; se encontró que en 16 entidades se requería dedicación a tiempo completo y en siete el requisito era capacitación previa.

El movimiento latinoamericano de Jóvenes Médicos de Familia (Waynakay) que aglutina especialistas con hasta cinco años de egresados y residentes de la especialidad, trabaja en la coordinación de pasantías en diferentes instituciones a través de las Asociaciones miembro de CIMF y las organizaciones de jóvenes médicos de familia de otras regiones de WONCA. Esta estrategia de pasantías que se encuentra afianzada, se identificó como un recurso valioso para promover y potenciar las pasantías específicas en investigación.

\section{Financiamiento de la investigación en Medicina Familiar}

Para obtener información sobre financiación, se encuestaron a 60 profesionales de la salud, en su mayoría médicos de familia, por medio de un instrumento virtual. Un 77\% de los encuestados tenían experiencia en investigación, la mayoría de ellos como investigadores principales. El percentil 75 para número de años de experiencia se ubicó en 15 (Rango 1-39). 
Cuadro 4. Organismos que ofrecen pasantías en investigación ( $n=26)$.

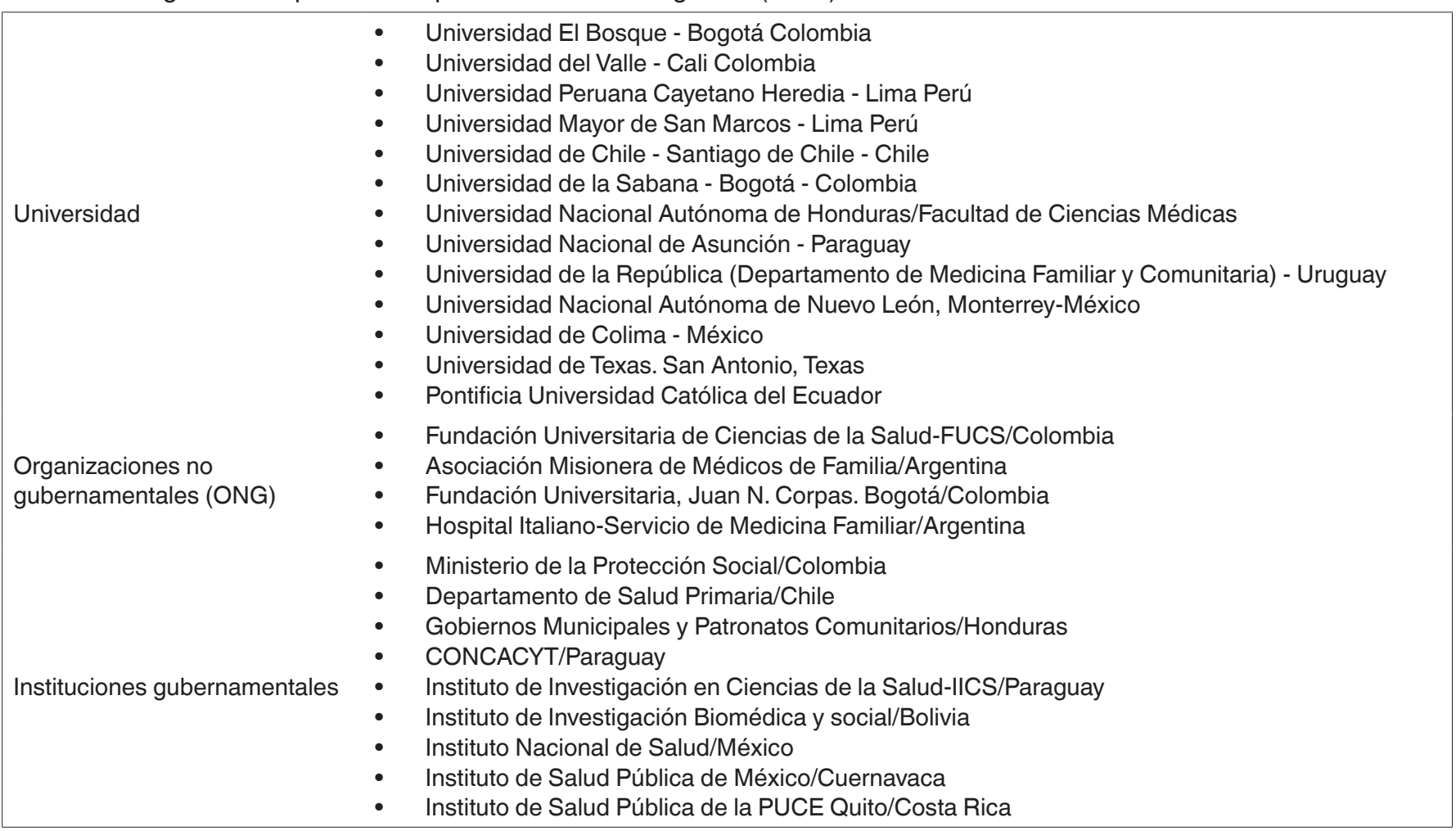

La mayoría de las investigaciones fueron financiadas con recursos propios del investigador (Gráfico 2). En los casos en que se identificó la presencia de una fuente financiadora externa al investigador, predominó el origen privado de los fondos, por encima de los fondos públicos. Las Universidades representaron $22 \%$ de las fuentes financiadoras.

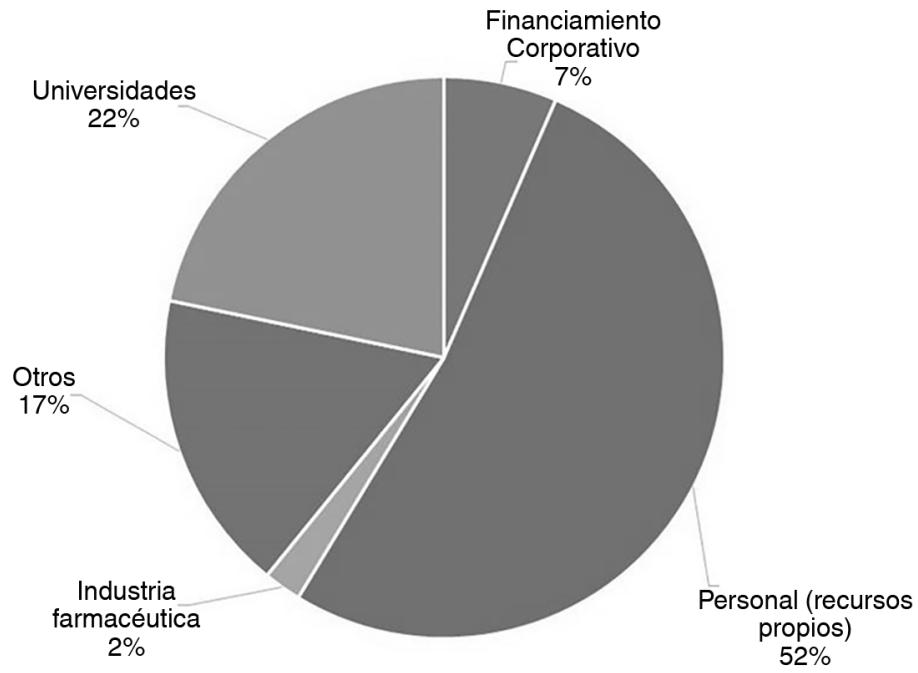

Gráfico 2. Financiamiento de la investigación en medicina familiar y comunitaria en una muestra no probabilística de investigadores activos en Iberoamérica $(n=60)$. 
La mayoría de los encuestados desconocía fuentes de financiamiento para la investigación y un $25 \%$ consideraba que existen trabas jurídicas para el acceso. La experiencia en la gestión de fondos de financiamiento fue evaluada en forma cualitativa. Se encontró satisfacción vinculada a la rapidez o sencillez de los trámites en una minoría de opiniones. En la mayoría se identificaron barreras y dificultades en esta gestión en distintos niveles: acceso (insuficiente priorización de la investigación en atención primaria), burocracia, impuestos, tiempo necesario para la gestión, retrasos en los desembolsos del dinero por las instituciones.

\section{Conclusiones}

Las Cumbres anteriores han definido estrategias cruciales para fortalecer la investigación en los diversos países y la región en su conjunto. Sin embargo, aún falta avanzar en la implementación de estas acciones, ya sea como colectivo regional o al interior de cada país en particular.

Surge como estrategia fundamental y primaria asegurar la comunicación y cooperación entre los países de Iberoamérica, mediante una definición de una agenda común, mancomunando esfuerzos, y el desarrollo de una plataforma compartida para divulgar información, proyectos, recursos, oportunidades, métodos y técnicas de investigación en atención primaria. IBIMEFA aparece como recurso fundamental para implementar esta estrategia.

La presencia de investigadores que trabajan activamente en líneas consideradas prioritarias por la región es una característica importante identificada en este relevamiento. Informa sobre la coherencia de los investigadores de la medicina familiar y comunitaria, que investigan en los problemas considerados prioritarios al tiempo que permite asignar una calificación inicial de pertinente a la investigación que se está desarrollando en el área.

Otro de los recursos identificados, fue la amplia gama de oportunidades para realizar pasantías en distintos centros gubernamentales y no gubernamentales de la región. Las universidades son los principales referentes para esta actividad.

Las limitaciones para obtener fuentes de financiamiento son barreras a superar a fin de fortalecer la investigación en MFYC en Iberoamérica. La voluntad de investigar, puesta en evidencia por el autofinanciamiento predominante como fuente de recursos en la región, debe acompañarse de la adquisición de conocimientos y competencias para la búsqueda de fondos. La Red IBIMEFA puede contribuir en gran medida a potenciar los recursos intrínsecos de la medicina familiar y comunitaria y sus asociaciones para la investigación, al tiempo que generar nuevos espacios y recursos por la coordinación y complementación. Mejorar las habilidades de investigación contribuirá a mejorar la captación de fondos para la producción de conocimiento en MFYC y atención primaria.

\section{Limitaciones de este estudio}

La principal debilidad identificada en este trabajo es el método de recolección de datos, no sistematizado, y el tipo de muestreo no probabilístico que limita la inferencia de los resultados. No obstante, el alto nivel de participación en número de personas y países con que contó este grupo de trabajo, y el número de respuestas recibidas desde personas designadas por las asociaciones de MFYC en cada país consideradas referentes en el tema, puede dotar de fortaleza a los datos, a pesar de las debilidades identificadas. Futuras intervenciones pueden ser diseñadas para ampliar los alcances de esta exploración al universo de investigadores o a una muestra representativa.

Casi $60 \%$ de los encuestados sobre líneas de investigación a priorizar no eran investigadores activos en ese momento. Identificado este dato, debe advertirse que las respuestas pueden reflejar líneas de investigación necesarias en la región, o también los temas donde se necesita mayor actualización en el conocimiento de la práctica clínica, lo cual podría resolverse por la investigación o no. No obstante esta consideración, la concordancia entre las líneas priorizadas y las líneas activas es un dato que aporta consistencia a los resultados. 


\section{Recomendaciones}

1. Continuar el trabajo para el desarrollo y fortalecimiento de la Red IBIMEFA como recurso para la integración de investigadores de la región; identificar y construir de oportunidades para la formación de investigadoes y el financiamiento de proyectos.

2. Mantener en forma activa y prospectiva la actualización del diagnóstico sobre situación de la investigación en Iberoamérica en forma participativa.

3. Establecer un registro formal de investigadores de la región, con datos que faciliten el intercambio de experiencias, la formación y el desarrollo de estudios colaborativos.

4. Avanzar en la identificación de líneas prioritarias de investigación con base en una cuidadosa convocatoria a los investigadores y referentes de la MFYC en cada país, los jóvenes MFYC y la comunidad, para enriquecer la lista de prioridades que se ha obtenido en esta primera fase de trabajo.

5. Desarrollar un proceso de gestión del financiamiento con representación internacional, que sirva de área consultora a los investigadores de MFYC.

6. Promover desde CIMF un diálogo más cercano con las Universidades y las Agencias Financiadoras como forma de contribuir a la generación de oportunidades en la Región Iberoamericana para el desarrollo de la Investigación en MFYC.

7. Incrementar los recursos y acciones para la comunicación y difusión dentro de CIMF, que favorezcan una mayor integración de los investigadores y difusión de la producción científica.

\section{Referencias}

1. Herrera JA. La investigación en medicina de familia en el siglo XXI (Editorial). Aten Primaria. 2008;40(9):435-6.

2. Van Weel C, Rosser WW. Improving health care globally: a critical review of necessity of family medicine research and recommendations to build research capacity. Ann FamMed. 2004;2(supl):s5-s16.

3. Herrera JA. Atención primaria y mortalidad materno-infantil en Iberoamérica. Aten Primaria. 2013;45(5):244-8.

4. Rubinstein A (coord). Investigación en la práctica de la medicina familiar:¿una causa perdida o un desafío pendiente? Colombia Médica. 2012;43(1). Disponible en: http://colombiamedica.univalle.edu.co/index.php/comedica/article/view/1065/1687

5. Fernández MA, Rojas G, Irigoyen A, Roo JB. Producción y difusión del conocimiento en Medicina Familiar en Iberoamérica. Rev Bras Med Fam Comunidade. 2016;12(Suppl 1):71-87.

6. Carta de Fortaleza. III Cumbre Iberoamericana de Medicina Familiar. 29 y 30 de abril de 2008; Fortaleza, Brasil. Disponible en: http://www.rbmfc.org.br/rbmfc/article/viewFile/432/356

7. Carta de Asunción. IV Cumbre Iberoamericana de Medicina Familiar. 15 y 16 de noviembre de 2011. Asunción, Paraguay. Dispoinible en: http://www.sbmfc.org.br/media/file/Carta\%20de\%20Asuncion.pdf

8. Carta de Quito. V Cumbre Iberoamericana de Medicina Familiar. 11 y 12 de abril de 2013. Quito, Ecuador. Disponible en: http://www.salud.gob.ec/carta-de-quito-v-cumbre-iberoamericana-de-medicina-familiar/

9. Red Iberoamericana de Medicina Familiar (IBIMEFA). Reporte del 2o Taller Iberoamericano de Medicina Familiar y Atención Primaria en Montevideo, marzo 18 de 2015. (Documento de Trabajo de la Confederación Iberoamericana de Medicina Familiar), Montevideo: Junio 2015. Disponible en: https://drive.google.com/file/d/OBOlGtVUOBhBMbEFSSG1TdENOU0k/view 